\title{
Infection Due to Vibrio Harveyi and Photobacterium Damselae Following Injury into a Marine Environment
}

\author{
Dimosthenis Chochlakis ${ }^{1}$, Sofia P Varveraki ${ }^{2}$, Dimitrios M Kostalas ${ }^{2}$, Chrysoula Koutsia Carouzou ${ }^{2}$ \\ and Anna Psaroulaki ${ }^{1 *}$
}

${ }^{1}$ Laboratory of Clinical Microbiology and Microbial Pathogenesis, Unit of Environmental Microbiology, School of Medicine, University of Crete, Greece

${ }^{2}$ General Hospital Asklepieio Voulas, Greece

*Corresponding author: Anna Psaroulaki, Faculty of Medicine, Laboratory of Clinical Microbiology and Microbial Pathogenesis,

University of Crete, Heraklion, Crete 71110, Greece

\section{ARTICLE INFO}

Received: 幽 October 03, 2019

Published: 幽October 18, 2019

Citation: Dimosthenis C, Sofia P V, Dimitrios M K, Chrysoula Koutsia C, Anna Psaroulaki. Infection Due to Vibrio Harveyi and Photobacterium Damselae Following Injury into a Marine Environment. Biomed J Sci \& Tech Res 22(2)-2019. BJSTR. MS.ID.003712.
ABSTRACT

Skin infections due to marine microorganisms following wounds into the water are not commonly reported. We report herein a case of wound infection, caused by Photobacterium damselae and by Vibrio harveyi, in a 44-year-old male patient, following a wound injury into the sea. Both bacteria were isolated by classical microbiological methods and identified using Matrix-Assisted Laser Desorption-Ionization Time of Flight Mass Spectrometry (MALDI-TOF) and PCR-sequencing analysis. The patient received all the necessary antibiotic regimen (cefuroxamine, teicoplanin, and amikacin) and recovered five days later. This is not the first case of concomitant infection from these two microorganisms following a skin injury. Clinicians should always have in mind such a possible infection since absence or late diagnosis may lead to necrotizing fasciitis.

\section{Introduction}

Vibrio harveyi is a Gram-negative, bioluminescent, marine bacterium in the genus Vibrio. It was originally described as Photobacterium fischeri [1], was later named after Beneckea harveyi [2] until it was given its final name, $V$. harveyi, by Baumann [3]. It is mainly found in aquatic environments, in tropical sea-waters and in mollusks [4]. Of the Vibrio species, the most frequently described infections in Europe are due to V. parahaemolyticus and V. vulnificus [5]. Photobacterium is a Gram negative bacterium belonging to the family Vibrionaceae. It was assigned its name at a re-classification that took place at the early 90's [6]. Photobacterium damselae is considered as being among the most virulent and is divided into two subspecies: piscicida and damsela. Photobacterium damselae subspecies piscicida is the causative agent of fish pasteurellosis which disease accounts for severe annual losses in fish farming enterprises. Photobacterium damselae subspecies damselae was formerly known as $V$. damsela. A number of human infections, following injury, by Vibrios other than $V$. harveyi have been reported; on the other hand a number of cases due to infection from P. damselae have been reported in the past [7-12]. To date, a single case of dual infection due to Photobacterium damselae and Vibrio

harveyi has been published [13]. In the current report, we present a case of human infection due to these aquatic species, following injury at a marine environment.

\section{Materials and Methods}

\section{Case Description}

A 44-year-old male patient, free of underlying diseases or travel history, was submitted at the department of orthopedics following an accident he suffered while doing wind surfing. The patient had an open right patella trauma following an overwhelming fracture (Gustillo III B), as noticed from a F/P Ro of the right knee. Immediately, two samples were received from the open wound with the use of cotton swabs and were sent for culture analysis. The samples were collected before the administration of any antibiotics. In the continuity, the trauma was washed followed by puncture and immobilization of the joint by placing a patellar splint. A number of laboratory and imaging tests were performed, including blood testing, triplex fast (-) and chest Ro; the patient was administered one dose of cefuroxamine $750 \mathrm{mg}$ and a surgery followed together 
with osteosynthesis of the patella fracture through the tension band technique. The patient was put under cefuroxamine $11500 \mathrm{mg}$ $\mathrm{x} 1$ /day for five days), teicoplanin (400mg x 2/day for five days) and amikacin (500mg x 2/day for two days); during hospitalization he did not show any systemic symptoms like fever, chills, and so forth. The post-surgery follows up was normal and the patient was discharged five days later with a change at his antibiotic regime (ciprofloxacin per os for seven days).

\section{Bacteriology}

The samples collected from the open wound were cultured on agar plates ((bioMérieux, Marcy l'Etoile, France) and in particular, on MacConkey agar, Sabouraud agar, Chapman agar (all three were incubated at aerobic conditions), chocholate agar (incubated under 5-10\% $\mathrm{CO}_{2}$ ), enrichment broth cooked meat and Columbia agar (incubated at anaerobic conditions). All plates were incubated at $37^{\circ} \mathrm{C}$

\section{MALDI-TOF Identification}

From each plate a number of distinctive colonies were selected, deposited on an MTP 96 target polished steel plate (Bruker Daltonics) and allowed to dry at room temperature. One $\mu$ l of a matrix solution (alpha-Cyano-4-hydroxycinnamic acid) (Bruker Daltonics) saturated with $2.5 \%$ trifluoroacetic acid and $50 \%$ acetonitrile was added and allowed to co-crystallize with each sample. Identifications were performed on a Microflex MALDI-TOF MS (Bruker Daltonics) using Flex Control software version 3.0 (build 184) and MALDI automation control version 2.0.43.8. Calibration of the MALDI-TOF MS software FlexControl was performed using peaks of Escherichia coli DH5 alpha (Bruker Bacterial Test Standard). The spectra were analysed in an $\mathrm{m} / \mathrm{z}$ range of 2.000-20.000. A reference spectrum for each strain was extracted based on the spectra generated. In order to identify the species of the isolates, the generated spectra were evaluated against the MALDI Biotyper database, containing a total of 73 reference spectra corresponding to 47 Vibrio species and five reference spectra corresponding to two Photobacterium species and two Photobacterium subspecies. Results were expressed as logscore values ranging from 0 to 3.0 , where values of $<1.7$ correspond to not reliable genus identification, 1.7-1.999 to probable genus identification, 2.0-2.299 to secure genus and probable species identification and 2.3-3.0 to highly probable species identification.

\section{Molecular Characterization}

DNA was extracted from isolates using the Tissue extraction kit (Qiagen, Hilden, Germany) according to the manufacturer's recommendations. DNA was kept at $-20^{\circ} \mathrm{C}$ until further analysis. $16 \mathrm{~s}$ rRNA sequencing was used for PCR amplifications, as previously described [14]. PCR products were purified (PCR product purification kit, Qiagen, Germany) and were directly sequenced using the sequencer CEQ 8000 Beckman Coulter (Bioanalytica - Genotype, Athens). All PCR products were sequenced twice to verify the revealed sequences. The sequences revealed were processed using Chromas v1.49 and Lasergene Ver.7.1 software for viewing the chromatograms and editing of the retrieved nucleotide sequences, and nucleotide BLASTn for investigating the homology of the obtained sequences compared to the NCBI databases.

\section{Susceptibility Testing}

Antibiotic susceptibility testing was performed through disk diffusion on Muller Hinton agar. Since the genus Photobacterium belongs to the family of Vibrionaceae, both isolates were tested as recommended by the Clinical and Laboratory Standard Institute (CLSI) for Vibrio species. Vibrio. harveyi was incubated at room temperature. Moreover, the susceptibility against amikacin was carried out by the use of E-testing.

\section{Result}

\section{Bacteriology}

No growth was recorded following the 24-hour incubation. Re-cultures were performed from the enrichment broth and 24 hours later medium sized isolates of light gray colour were grown on MacConkey and Columbia agar. The isolates were identified as $P$. damselae. All plates were kept at room temperature for another 24 hours during which, slight $\beta$-haemolysis around the gray isolates was observed, while slow growing isolates (flat, small sized, yellow colour) were grown on Columbia agar. These isolates were identified as $V$. harveyi. Both bacteria species were oxidase positive, catalase negative, gram negative. The identification was performed via the automated system Phoenix 2 (Becton Dickinson) using the API 20E (BioMerieux) and was confirmed by MADLI-TOF MS.

\section{MALDI-TOF Identification}

Photobacterium damselae and V. harveyi were identified at scores 2.218 and 2.167 respectively. Results are given in Table 1.

Table 1: MALDI-TOF identification results obtained for $P$. damselae and $V$. harveyi. The best 10 hits, as provided by the software, are presented. Results were expressed as log-score values ranging from 0 to 3.0 , where values of $<1.7$ correspond to not reliable genus identification, 1.7-1.999 to probable genus identification, 2.0-2.299 to secure genus and probable species identification and 2.3-3.0 to highly probable species identification.

\begin{tabular}{|c|c|}
\hline Matched Pattern & Score value \\
\hline \multicolumn{2}{|l|}{ Vibrio Species } \\
\hline Vibrio harveyi DSM 19623T DSM & 2.167 \\
\hline Vibrio parahaemolyticus DSM 10027T DSM & 1.824 \\
\hline Vibrio parahaemolyticus DSM 15477 DSM & 1.794 \\
\hline Vibrio parahaemolyticus DSM 11058 DSM & 1.761 \\
\hline Vibrio harveyi LMG 19643 HAM & 1.737 \\
\hline Vibrio harveyi LMG 4044T HAM & 1.733 \\
\hline Vibrio parahaemolyticus DSM 11058 DSM & 1.697 \\
\hline Vibrio harveyi LMG 7890 HAM & 1.679 \\
\hline Vibrio parahaemolyticus CCM 5937 CCM & 1.66 \\
\hline Vibrio alginolyticus CCM 7037 CCM & 1.653 \\
\hline \multicolumn{2}{|l|}{ Photobacterium Species } \\
\hline
\end{tabular}




\begin{tabular}{|c|c|}
\hline $\begin{array}{c}\text { Photobacterium damselae ssp piscicida ATCC } \\
\text { 29690 EGS }\end{array}$ & 2.218 \\
\hline $\begin{array}{c}\text { Photobacterium damselae ssp piscicida PC846_1 } \\
\text { EGS }\end{array}$ & 2.192 \\
\hline $\begin{array}{c}\text { Photobacterium damselae ssp damselae DSM } \\
\text { 7482T HAM }\end{array}$ & 2.172 \\
\hline $\begin{array}{c}\text { Photobacterium damselae ssp damselae CDC } \\
\text { 2227_81 EGS }\end{array}$ & 1.699 \\
\hline Kocuria rosea IMET 11363T HKJ & 1.415 \\
\hline Enterobacter cloacae 20105_2 CHB & 1.315 \\
\hline Escherichia coli ESBL_EA_RSS_1528T CHB & 1.308 \\
\hline Arthrobacter ilicis DSM 20138T DSM & 1.259 \\
\hline Pseudomonas tolaasii LMG 2342T HAM & 1.244 \\
\hline Proteus mirabilis DSM 30115 DSM & 1.237 \\
\hline
\end{tabular}

\section{Molecular Characterization}

The DNA sequencing analysis of the positive PCR products revealed $100 \%$ similarities to already published sequences of $V$. harveyi (AB477515) and P. damselae subspecies damselae (AJ535853).

\section{Susceptibility Testing}

Both P. damselae and V. harveyi were susceptible to all antibiotics they were tested against, with the exception of ampicillin as shown on Table 2.

Table 2: Antibiotic susceptibility testing by disk diffusion according to the CLSI guidelines. Amikacin was tested by the use of E-testing. R: resistant. S: sensitive.

\begin{tabular}{|c|c|c|}
\hline Antibiotic substance & P. Damselae & V. Harveyi \\
\hline Ampicillin & $\mathrm{R}$ & $\mathrm{R}$ \\
\hline Amixicillin/Clavulanic acid & $\mathrm{S}$ & $\mathrm{S}$ \\
\hline Piperacillin/Tazobactam & $\mathrm{S}$ & $\mathrm{S}$ \\
\hline Imipenem & $\mathrm{S}$ & $\mathrm{S}$ \\
\hline Meropenem & $\mathrm{S}$ & $\mathrm{S}$ \\
\hline Cefoxitin & $\mathrm{S}$ & $\mathrm{S}$ \\
\hline Cefotaxime & $\mathrm{S}$ & $\mathrm{S}$ \\
\hline Cotrimoxazole & $\mathrm{S}$ & $\mathrm{S}$ \\
\hline Gentamicin & $\mathrm{S}$ & $\mathrm{S}$ \\
\hline Amikacin & $\mathrm{S}$ & $\mathrm{S}$ \\
\hline Tetracycline & $\mathrm{S}$ & $\mathrm{S}$ \\
\hline Ciprofloxacin & $\mathrm{S}$ & $\mathrm{S}$ \\
\hline
\end{tabular}

\section{Discussion}

Two cases of human infection due to $V$. harveyi and $P$. damselae have been reported so far [13-15]. Both these cases were described in Australia; none of these patients had any significant underlying medical history that could jeopardize the whole situation. In all three cases (the two in Autstralia and the one described herein) the infection by these bacteria was associated with wound into seawater. In the Australian cases, a haemorrhagic bullous lesion surrounded by erythema and swelling of the hand or a necrotic area were recorded but these findings could be attributed to the late hospital visit. In our case no such or any similar findings were observed since the patient was admitted at the hospital right after the injury he suffered. All three cases were prescribed either quinolones or doxycycline and all three had a positive outcome.

Generally, Vibrios are opportunist microorganisms that only cause disease when the host organisms are immune-suppressed or otherwise physiologically stressed. Nevertheless, a number of studies have been carried out world-wide on Vibrio infections, coming to a conclusion that wound infections caused by marine bacteria appear to be increasing in frequency and recognition. Many require some underlying syndrome, most commonly involving liver disease, but others appear able to infect fully immunocompetent and healthy individuals. Some Vibrio species are potentially fatal and require rapid and aggressive treatment [16]. In the USA, almost 8000 people suffer from disease caused by Vibrio species each year. Of these infections, 5200 are foodborne in origin, while around 2800 are caused by non-foodborne Vibrio infections (NFVIs) [17]. In a large study carried out from 1997 through 2006, 1210 (25\% of the total infections) cases were attributed to NFVIs. Of the species isolated, the most frequent was V. vulnificus, followed by V. alginolyticus and V. parahemolyticus; other Vibrio species caused infections at lesser extents. All Vibrio species were isolated either from a wound or from another source with documented direct contact with saltwater, marine wildlife, raw seafood, or seafood drippings.

The majority of infections occurred from May through September, while few infections were also recorded during winter months. Of the 369 total deaths occurring due to any Vibrio speciesassociated illness, NFVIs accounted for 79 (21\%). Searching the bibliography, we discovered that the first reported presence of Vibrio species in Greek sea waters goes back to the mid-70s [18]. From that time, a number of cases attributed to infections due to Vibrio species, other than $V$. harveyi, following injury, have been reported in Greece, such as $V$. alginolyticus causing suppurative chronic otitis media in a Greek diver [19], V. vulnificus causing infection of the lower extremities [20,21]. Most, but not all, patients suffered from some kind of immuno-suppression and/or some kind of underlying disease. Generally, almost all persons who develop Vibrio wound infections do so following infection of a pre-existing wound or one obtained while engaged in recreational or fishing activities in coastal waters [22].

It has been suggested that global climate change resulting in increasing water temperature and flooding of low-lying coastal areas due to rising sea levels may be regarded as important factors in the proliferation of many water-borne infectious diseases. Increases in seawater temperature of $4-5^{\circ} \mathrm{C}$ in Southern Europe and in the Black Sea region, and increases in an annual mean temperature increase in the order of $2.5-3.5^{\circ} \mathrm{C}$ in Western Europe are expected to be recorded over the next decades, resulting in an increase in the risk of proliferation and on infection due to waterborne pathogens such as Vibrio species [5]. 
As regards $P$. damselae, a number of human infections following wound injuries have been described so far [7-12]. In the majority of the cases, humans suffered from severe infection, particularly in the form necrotizing fasciitis, while at least two patients had a fatal outcome. In experiments carried out in fish, it has been shown that the pathogen can be transmitted through water, with the spread of the disease being dependent on temperature and water salinity [23]. Perhaps the pathogen can use similar mechanisms to penetrate human skin especially in cases of wound injury, even if this is a minor one. To our knowledge, no such case of Photobacterium infection following some kind of injury has been reported in Greece so far.

\section{Conclusion}

Physicians should be aware of infections, either single or dual, due to microorganisms found in marine environments in case of patients presenting with wound infections and reporting contact to seawater and fish or exposure to seafood.

\section{Acknowledgement}

None.

\section{Conflict of Interest}

No conflict of interest.

\section{References}

1. Beijerinck MW (1889) Le Photobacterium luminosum, Bacterie lumineuse de la Mer du Nord. Arch Neerl des Sci Exact et Nat 23: 401427.

2. Reichelt JL, Baumann P (1973) Taxonomy of the marine, luminous bacteria. Arch Mikrobiol 94(4): 283-330.

3. Baumann P, Baumann L, Bang SS, Woolkalis MJ (1980) Reevaluation of the taxonomy of Vibrio, Beneckea, and Photobacterium: abolition of the genus Beneckea. Curr Microbiol 4(3): 127-132.

4. Owens L, Busico Salcedo N, Vibrio harveyi (2006) Pretty Problems in Paradise. In Thompson F, Austin B, Swings J (Eds.). The Biology of Vibrios, ASM Press, USA.

5. Baker Austin C, Stockley L, Rangdale R, Martinez Urtaza J (2010) Environmental occurrence and clinical impact of Vibrio vulnificus and Vibrio parahaemolyticus: a European perspective. Environ Microbiol Rep 2(1): 7-18.

6. Smith SK, Sutton DC, Fuerst JA, Reichelt JL (1991) Evaluation of the genus Listonella and reassignment of Listonella damsela (Love et al.) Mac Donell and Colwell to the genus Photobacterium as Photobacterium damsela comb. nov. with an emended description. Int J Syst Bacteriol 41(4): 529-534.
7. Aigbivbalu L, Maraqa N (2009) Photobacterium damsela wound infection in a 14-year-old surfer. South Med J 102(4): 425-426.

8. Clarridge JE, Zighelboim Daum S (1985) Isolation and characterization of two hemolytic phenotypes of Vibrio damsela associated with a fatal wound infection. J Clin Microbiol 21(3): 302-306.

9. Dryden M, Legarde M, Gottlieb T, Brady L, Ghosh HK (1989) Vibrio damsela wound infections in Australia. Med J Aust 151(9): 540-541.

10. Morris JG, Miller HG, Wilson R, Tacket CO, Hollis DG, et al. (1982) Illness caused by Vibrio damsela and Vibrio hollisae. Lancet 1(8284): 12941297.

11. Perez Tirse J, Levine JF, Mecca M (1993) Vibrio damsela. A cause of fulminant septicemia. Arch Intern Med. 153(15): 1838-1840.

12. Yuen KY, Ma L, Wong SS, Ng WF (1993) Fatal necrotizing fasciitis due to Vibrio damsela. Scand J Infect Dis 25(5): 659-661.

13. Hundenborn J, Thurig S, Kommerell M, Haag H, Nolte O (2013) Severe Wound Infection with Photobacterium damselae ssp. damselae and Vibrio harveyi, following a Laceration Injury in Marine Environment: A Case Report and Review of the Literature. Case Rep Med 2013: 610632.

14. Lane L (1991) 16S/23S sequencing. In: Stackebrandt E, Goodfellow M, editors. Nucleic Acid Techniques in Bacterial Systematics. John Wiley \& Sons, Chichester, UK, pp. 115-176.

15. Akram A, Stevens RP, Konecny P (2015) Photobacterium damselae and Vibrio harveyi hand infection from marine exposure. Med J Aust 203(5): 224-225.

16. Oliver JD (2005) Wound infections caused by Vibrio vulnificus and other marine bacteria. Epidemiol Infect. 133(3): 383-391.

17. Dechet AM, Yu PA, Koram N, Painter J (2008) Nonfoodborne Vibrio infections: an important cause of morbidity and mortality in the United States, 1997-2006. Clin Infect Dis 46(7): 970-976.

18. Papadakis JA, Trichopoulos D, Papoutsakis G, Kalapothaki V, Vassiliadis $P$ (1975) Isolation of water vibrios in Greece (author's transl). Ann Microbiol (Paris)126(3): 361-365.

19. Tsakris A, Psifidis A, Douboyas J (1995) Complicated suppurative otitis media in a Greek diver due to a marine halophilic Vibrio sp. J Laryngol Otol 109(11): 1082-1084.

20. Mouzopoulos G, Stamatakos M, Tzurbakis M, Batanis G, Michou E, et al. (2008) Lower extremity infections by vibrio vulnificus. Chirurgia (Bucur) 103(2): 201-203.

21. Thodis E, Kriki P, Kakagia D, Passadakis P, Theodoridis M, et al. (2009) Rigorous Vibrio vulnificus soft tissue infection of the lower leg in a renal transplant patient managed by vacuum therapy and autologous growth factors. J Cutan Med Surg 13(4): 209-214.

22. Howard R, Brennaman B, Lieb S (1986) Soft tissue infections in Florida due to marine vibrio bacteria. J Fla Med Assoc 73(1): 29-34.

23. Fouz B, Toranzo AE, Milan M, Amaro C (2000) Evidence that water transmits the disease caused by the fish pathogen Photobacterium damselae subsp. damselae. J Appl Microbiol 88(3): 531-535. 
ISSN: 2574-1241

DOI: 10.26717/BJSTR.2019.22.003712

Anna Psaroulaki. Biomed J Sci \& Tech Res

(c) (i) This work is licensed under Creative BY Commons Attribution 4.0 License

Submission Link: https://biomedres.us/submit-manuscript.php

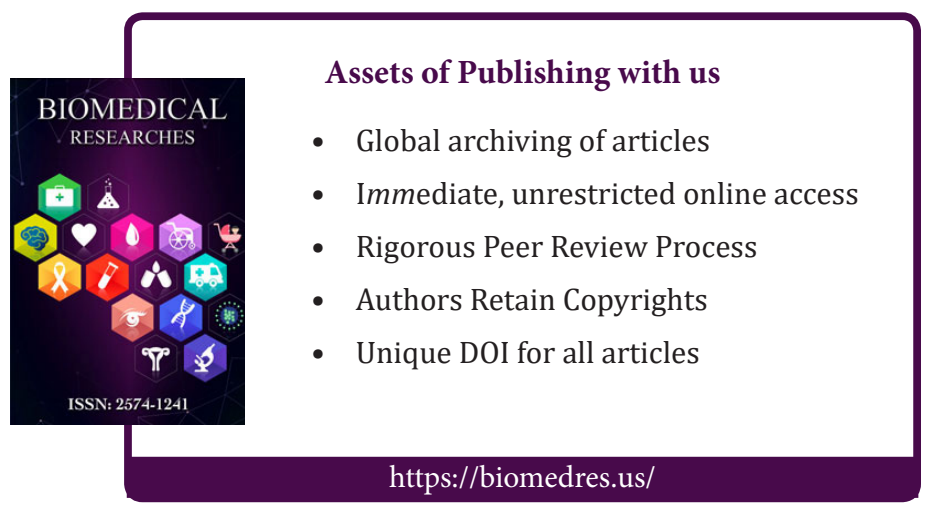

